\title{
Equivalence of stationary state ensembles
}

\author{
H.J. Hilhorst and F. van Wijland \\ Laboratoire de Physique Théorique ${ }^{1}$ \\ Bâtiment 210, Université de Paris-Sud \\ 91405 Orsay Cedex, France
}

October 28, 2018

\begin{abstract}
We show that the contact process in an ensemble with conserved total particle number, as simulated recently by Tomé and de Oliveira [Phys. Rev. Lett. 86 (2001) 5463], is equivalent to the ordinary contact process, in agreement with what the authors assumed and believed. Similar conserved ensembles and equivalence proofs are easily constructed for other models.

PACS 02.50.Ga, 05.10.Ln
\end{abstract}

LPT ORSAY 01/83

${ }^{1}$ Laboratoire associé au Centre National de la Recherche Scientifique - UMR 8627 
A statistical mechanical ensemble is a probability distribution on a set of microstates. For systems in thermodynamic equilibrium the ensemble is known a priori. The equivalence of the standard equilibrium ensembles (microcanonical, canonical, ... ) is a well-known fact. In Monte Carlo simulations an easy way to generate a given ensemble is to impose detailed balancing on the transition rates between the microstates. A system of statistical physics may be defined more generally, and more implicitly, not by an a priori given ensemble but by the transition rates between the microstates. The stationary (= time independent) solution of the resulting stochastic equation of motion then constitutes an ensemble which, unlike the standard equilibrium ensembles, cannot usually be expressed in explicit analytic form. Such stationary ensembles [1] arise, e.g., in reaction-diffusion type systems, of which one example is the object of study here.

We consider the contact process (CP) introduced by Harris [2], which takes place on an infinite regular lattice each of whose sites may be empty or occupied by a particle. The transition rates are such that particles (i) are created on an empty site at a rate $n_{\mathrm{nn}} / z$, where $n_{\mathrm{nn}}$ is the number of occupied nearest neighbors of that site and $z$ the lattice coordination number; and (ii) annihilate spontaneously and independently at a rate $k$.

The contact process is of interest because its stationary ensemble exhibits a phase transition between an active state (particles present) and an absorbing state (empty lattice) for a certain critical value $k=k_{c}$ of the annihilation rate [3]. Monte Carlo simulations of the active state on finite lattices and for $k$ close to $k_{c}$ suffer from the complication that in any long enough run there will inevitably occur a fluctuation that pushes the system irreversibly into the absorbing state. Various procedures have been proposed for dealing with this "accidental death", which occurs similarly in other model systems with transitions to absorbing states. Very recently Tomé and de Oliveira 㧮 had recourse to an elegant method, used earlier in a different context by Ziff and Brosilow [5], and which circumvents the accidental death problem altogether. Tomé and de Oliveira replace the ordinary contact process (CP) by a version with strictly conserved particle number $N$. In this conserved contact process (CCP) particles (i) are created at the same rate as in the $\mathrm{CP}$; but (ii) every creation is accompanied by the simultaneous annihilation of another particle picked at random on the lattice. It is our purpose here to show that the conserved ensemble $(\mathrm{CCP})$ is equivalent to the nonconserved ensemble $(\mathrm{CP})$. This equivalence was assumed and believed to be true by the authors [4], who nevertheless feel the need to invoke their results as an additional argument. The proof is simple and, just like the conserved ensemble method itself, can easily be adapted to other problems.

Let $j$ denote a lattice site and let $n_{j}$ be its occupation number $\left(n_{j}=0\right.$ or $=1$ ). It will be convenient to work with "spins" $s_{j}=2 n_{j}-1$, so that $s_{j}=$ \pm 1 . The symbol $s \equiv\left\{s_{j}\right\}$ then represents the set of occupation numbers. We associate [6] with each $s$ a basis vector $|s\rangle$ and with the time dependent 
probability distribution $P(s, t)$ the state vector

$$
|P\rangle_{t}=\sum_{s} P(s, t)|s\rangle
$$

The master equation for $P(s, t)$ is then equivalent to the evolution equation

$$
\frac{\mathrm{d}|P\rangle_{t}}{\mathrm{~d} t}=\mathcal{W}|P\rangle_{t}
$$

for the state vector, in which the "master operator" $\mathcal{W}$ is the infinitesimal generator of the transitions. The $s_{j}= \pm 1$ may be considered as the eigenvalues of a Pauli spin matrix $\sigma_{j}^{z}$. Since $\sigma_{j}^{x}$ reverses $s_{j}$, one may express $\mathcal{W}$ in terms of the $\sigma_{j}^{x}$ and $\sigma_{j}^{z}$ [0]. For the standard CP with annihilation rate $k$ this yields $\mathcal{W}=\mathcal{W}^{\mathrm{CP}}$ with

$$
\begin{aligned}
\mathcal{W}^{\mathrm{CP}} & =\mathcal{W}_{\text {cre }}+k \mathcal{W}_{\text {ann }} \\
\mathcal{W}_{\text {cre }} & =\sum_{j} \sum_{\delta}^{\prime}\left(\sigma_{j}^{x}-1\right) \frac{1-\sigma_{j}^{z}}{2} \frac{1+\sigma_{j+\delta}^{z}}{2} \\
\mathcal{W}_{\mathrm{ann}} & =\sum_{j}\left(\sigma_{j}^{x}-1\right) \frac{1+\sigma_{j}^{z}}{2}
\end{aligned}
$$

where $j+\delta$ is a neighbor site of $j$ and $\sum_{\delta}^{\prime}$ denotes $1 / z$ times the sum on all neighboring sites. For the CCP with exactly $N$ particles one finds $\mathcal{W}=\mathcal{W}^{\mathrm{CCP}}$ with

$$
\mathcal{W}^{\mathrm{CCP}}=\frac{1}{N} \sum_{j} \sum_{\delta}^{\prime} \sum_{i}\left(\sigma_{j}^{x} \sigma_{i}^{x}-1\right) \frac{1-\sigma_{j}^{z}}{2} \frac{1+\sigma_{j+\delta}^{z}}{2} \frac{1+\sigma_{i}^{z}}{2}
$$

(in which the term $i=j$ is identically zero). The question is now to demonstrate the equivalence, in a sense to be appropriately defined, of $\mathcal{W}^{\mathrm{CP}}$ and $\mathcal{W}^{\mathrm{CCP}}$. Our proof proceeds by two steps. The first one is to rewrite expression (6) for the operator $\mathcal{W}^{\mathrm{CCP}}$ by means of the substitution

$$
\sigma_{j}^{x} \sigma_{i}^{x}-1=\left(\sigma_{j}^{x}-1\right)+\left(\sigma_{i}^{x}-1\right)+\left(\sigma_{j}^{x}-1\right)\left(\sigma_{i}^{x}-1\right)
$$

Using that in the conserved ensemble $N=\sum_{i}\left(1+\sigma_{i}^{z}\right) / 2$ we find

$$
\mathcal{W}^{\mathrm{CCP}}=\mathcal{W}_{\text {cre }}+\mathcal{W}_{\mathrm{ann}} \kappa+\mathcal{W}_{\mathrm{R}}
$$

Here $\kappa=\mathcal{N}_{10} / N$ in which

$$
\mathcal{N}_{10}=\sum_{j} \sum_{\delta} \frac{1-\sigma_{j}^{z}}{2} \frac{1+\sigma_{j+\delta}^{z}}{2}
$$

is the operator for the total number of pairs of neighboring sites of which one is occupied and the other empty; and the "remainder" $\mathcal{W}_{\mathrm{R}}$ is given by

$$
\mathcal{W}_{\mathrm{R}}=\frac{1}{N} \sum_{j} \sum_{\delta}^{\prime} \sum_{i}\left(\sigma_{j}^{x}-1\right)\left(\sigma_{i}^{x}-1\right) \frac{1-\sigma_{j}^{z}}{2} \frac{1+\sigma_{j+\delta}^{z}}{2} \frac{1+\sigma_{i}^{z}}{2}
$$


We remark that whereas $\mathcal{W}_{\text {cre }}$ and $\mathcal{W}_{\text {ann }}$ are themselves master operators (i.e. correspond to master equations), the remainder $\mathcal{W}_{\mathrm{R}}$ is not; the reason is that although it conserves the total probability, it does not conserve the positivity of an initial probability distribution.

The second step of the proof is to consider the time evolution of the ensemble average of an arbitrary product $f \equiv \sigma_{\ell_{1}}^{z} \sigma_{\ell_{2}}^{z} \ldots \sigma_{\ell_{r}}^{z}$, knowing that all physical observables are linear combinations of such products. Let $\langle f\rangle_{t}^{\mathrm{CCP}}$ denote the average of $f$ in the conserved ensemble. It may be calculated as the scalar product

$$
\langle f\rangle_{t}^{\mathrm{CCP}}=\langle O|f| P\rangle_{t}
$$

where $|P\rangle_{t}=\exp \left(\mathcal{W}^{\mathrm{CCP}} t\right)|P\rangle_{0}$ and where the "projection state" $\langle O|$ is defined by $\langle O|=\sum_{s}\langle s|$ with the sum running on all $s$ irrespective of the number of particles present. From Eqs. (11) and (8) it follows that

$$
\frac{\mathrm{d}\langle f\rangle_{t}^{\mathrm{CCP}}}{\mathrm{d} t}=\left\langle f \mathcal{W}_{\mathrm{cre}}\right\rangle_{t}^{\mathrm{CCP}}+\left\langle f \mathcal{W}_{\mathrm{ann}} \kappa\right\rangle_{t}^{\mathrm{CCP}}+\left\langle f \mathcal{W}_{\mathrm{R}}\right\rangle_{t}^{\mathrm{CCP}}
$$

In the usual nonconserved ensemble one has in the same way from Eqs. (11) and (3) that

$$
\frac{\mathrm{d}\langle f\rangle_{t}^{\mathrm{CP}}}{\mathrm{d} t}=\left\langle f \mathcal{W}_{\mathrm{cre}}\right\rangle_{t}^{\mathrm{CP}}+k\left\langle f \mathcal{W}_{\mathrm{ann}}\right\rangle_{t}^{\mathrm{CP}}
$$

We now compare Eqs. (12) and (13). In the limit of an infinite system (that is statistically invariant under translations) the operator $\kappa$ will have vanishingly small fluctuations around its average $\langle\kappa\rangle_{t}^{\mathrm{CCP}}=\left\langle\mathcal{N}_{10}\right\rangle_{t}^{\mathrm{CCP}} / N$. Hence we may replace $\kappa$ by this average. Next we will show that the last term on the RHS of Eq. (12) vanishes in the limit of an infinitely large system. It is not sufficient for that to invoke the factor $1 / N$ in expression (10) for $\mathcal{W}_{\mathrm{R}}$. Instead, we will make the average $\left\langle f \mathcal{W}_{\mathrm{R}}\right\rangle_{t}^{\mathrm{CCP}}$ fully explicit. In order to do so we commute the $\sigma_{j}^{x}$ through $f$ using that $\left[\sigma_{j}^{z}, \sigma_{j}^{x}\right]_{+}=0$, and use that $\langle O| \sigma_{j}^{x}=\langle O|$. This gives

$$
\left\langle f \mathcal{W}_{\mathrm{R}}\right\rangle_{t}^{\mathrm{CCP}}=\frac{4}{N} \sum_{p=1}^{r} \sum_{q=1}^{r} \sum_{\delta}^{\prime}\left\langle f \frac{1-\sigma_{\ell_{p}}^{z}}{2} \frac{1+\sigma_{\ell_{p}+\delta}^{z}}{2} \frac{1+\sigma_{\ell_{q}}^{z}}{2}\right\rangle_{t}^{\mathrm{CCP}}
$$

The RHS of this expression involves a sum on $r^{2}$ terms and the summand makes no reference to the system size. Therefore it is now clear that in view of the prefactor $1 / N$ the average (14) vanishes in the limit of infinite system size. Hence Eq. (12) may be replaced with

$$
\frac{\mathrm{d}\langle f\rangle_{t}^{\mathrm{CCP}}}{\mathrm{d} t}=\left\langle f \mathcal{W}_{\text {cre }}\right\rangle_{t}^{\mathrm{CCP}}+\langle\kappa\rangle_{t}^{\mathrm{CCP}}\left\langle f \mathcal{W}_{\mathrm{ann}}\right\rangle_{t}^{\mathrm{CCP}}
$$

Comparison of (13) and (15) shows that any spin average evolves according to the same equations of motion in both ensembles provided we have $\langle\kappa\rangle_{t}^{\mathrm{CCP}}=k$, i.e.

$$
\left\langle\mathcal{N}_{10}\right\rangle_{t}^{\mathrm{CCP}} / N=k
$$


This equation relates the CP parameter $k$ to a time dependent CCP average. It can be satisfied only if that average is time independent, hence equal to its stationary state value. Therefore the condition for (13) and (12) to be equivalent becomes

$$
\left\langle\mathcal{N}_{10}\right\rangle_{\text {stat }}^{\mathrm{CCP}} / N=k
$$

where the average is on the stationary CCP ensemble. In the stationary state of the CCP, or for fluctuations around it that in the large- $N$ limit affect $\left\langle\mathcal{N}_{10}\right\rangle_{\text {stat }}^{\mathrm{CCP}} / N$ negligibly, equality (17) guarantees that the two averages $\langle f\rangle_{t}^{\mathrm{CP}}$ and $\langle f\rangle_{t}^{\mathrm{CCP}}$ obey the same equations. It follows in particular that for any $f$ we have $\langle f\rangle_{\text {stat }}^{\mathrm{CP}}=\langle f\rangle_{\mathrm{stat}}^{\mathrm{CCP}}$. This establishes the equivalence of the two stationary ensembles. A notable case which on the basis of the preceding discussion is excluded from the equivalence, is the relaxation towards equilibrium of an initial state in which all particles occupy random positions: In such a nonequilibrium process [1] the average of $\mathcal{N}_{10}$ is not constant (in either ensemble) and (17) cannot be satisfied.

The equivalence condition (17) derived here was introduced by Tomé and de Oliveira (Eq. (3) of [4]), who exploit it to determine the critical behavior of the contact process. Here we have furnished the proof (albeit a physicist's one) that their procedure was correct.

The construction of conserved ensembles by simultaneous execution of elementary transitions at uncorrelated lattice locations can easily be adapted to other models of interest in statistical physics. The same is true for the equivalence proof of this note.

\section{References}

[1] The more general stationary ensembles are referred to in Ref. [4] by the term "nonequilibrium". Here we prefer to reserve that term for time dependent ensembles.

[2] T.E. Harris, Ann. Probab. 2 (1974) 969.

[3] The transistion is in the important "Directed Percolation" universality class.

[4] T. Tomé and M.J. de Oliveira, Phys. Rev. Lett. 86 (2001) 5643.

[5] R. M. Ziff and B.J. Brosilow, Phys. Rev. A 46 (1992) 4630.

[6] L.P. Kadanoff and J. Swift, Phys. Rev. 165 (1968) 310.

[7] Such an expression for a master operator was first proposed and studied by B.U. Felderhof, Rep. Math. Phys. 1 (1971) 215; 2 (1971) 151. 